François HeEneN

UNIVERSITÉ D’ISLANDE

\title{
Mémoire et temps du passé
}

\section{Introduction}

E xpliquer la multiplicité des formes grammaticales du verbe utilisées dans une langue pour communiquer la référence temporelle passée d'un événement est un des objectifs de la sémantique et de la pragmatique. Lapproche qui sera utilisée dans cet article consistera à mettre en relation les temps grammaticaux du passé en français avec deux modes de travail de la mémoire. Dans un de ces modes la mémoire serait dirigée par une tâche définie et dans l'autre elle aurait libre cours et serait spontanée. L’imparfait serait corrélé à la mémoire spontanée, alors que le passé simple le serait à la mémoire à tâche positive. L'article sera organisé de la manière suivante. Nous commencerons par un aperçu sur les propriétés temporelles et aspectuelles principales du passé simple et de l'imparfait. La section suivante servira à présenter les principes fondamentaux de la théorie modulaire de l'esprit ainsi que les applications de cette théorie dans le domaine de la sémantique procédurale, après quoi l'hypothèse de cet article sera expliquée dans le cadre de ces deux théories. Ensuite viendront deux sections dans lesquelles nous verrons comment cette approche modifie notre conception des propriétés temporelles et aspectuelles du passé simple et de l'imparfait. La section 7 montrera qu'on peut justifier le lien entre imparfait et mémoire spontanée en se référant aux recherches sur le réseau cérébral par défaut. Deux sections seront ensuite consacrées respectivement au passé composé et au plus-queparfait dans lesquelles nous examinerons en quoi le critère du travail de la mémoire permet de comprendre la propriété commune à ces 
deux temps d'impliquer un état résultant de l'événement. Pour le passé composé, il sera proposé que l'état résultant est une implication faite sur base d'un épisode du passé que le locuteur a cherché à récupérer en tenant compte de sa pertinence pour l'interlocuteur. Ce serait la raison pour laquelle cette implication est ressentie comme «vraie à $S$ ». Pour le plus-que-parfait, l'état résultant sera vu comme une opération que le locuteur choisit lui-même de faire pour se représenter un épisode différent de celui décrit par l'énoncé. Dans la dernière section du corps de cet article, une tentative sera faite pour expliquer comment les quatre temps du passé du français, passé simple, imparfait, plus-que-parfait et passé composé, ont acquis des fonctions différentes, toutes liées au fonctionnement de la mémoire épisodique.

\section{Aperçu sur les analyses du passé simple et de l'imparfait}

Dans la présente section, nous allons passer en revue les propriétés temporelles et aspectuelles principales du passé simple et de l'imparfait qui ont été relevées par les analyses précédentes. ${ }^{1}$

Le passé simple a une valeur de passé incontournable puisqu'il est impossible de l'utiliser pour faire référence à des événements présents ou futurs. Cette valeur est plus constante que pour le passé composé, qui dans certains énoncés est compatible avec des adverbes indiquant le présent, et pour l'imparfait qui dans certaines structures de phrases est incompatible avec des adverbes indiquant le passé :

1. Maintenant, j'ai compris.

2. Si tu venais *hier je serais content.

La différence peut être due au fait que le passé simple est exclusivement utilisé dans des narrations où il fait toujours référence à des événements du passé, les autres temps ayant des usages plus variés.

Sur le plan aspectuel, on a constaté que le passé simple n’imposait pas de vue interne de l'action comme l'imparfait :

1 Parmi ces analyses, notons spécialement Arie L. Molendijk, Le passé simple et l'imparfait : une approche reichenbachienne, Amsterdam/ Atlanta, Rodopi, 1990; et Carl Vetters, « Passé simple, et imparfait : un couple mal assorti », Langue française, 100/1993, Temps et aspect dans la langue française, pp. 14-30. 
3. Il regarda la vitrine.

4. Il regardait la vitrine.

L'exemple à l'imparfait fait voir le sujet regardant la vitrine. On l'imagine dans la rue se tenant debout devant un magasin, son regard dirigé vers un étalage. Dans une série d'articles consacrés à l'imparfait, l'hypothèse a été avancée que ces images mentales étaient générées par la mémoire encyclopédique à laquelle l'imparfait offre un accès direct. ${ }^{2}$ En comparaison, la phrase au passé simple n'impose pas de se représenter la scène, mais elle ne l'interdit pas non plus.

Une autre particularité aspectuelle du passé simple est son incompatibilité avec des énoncés où une des bornes de l'événement est intentionnellement cachée. ${ }^{3}$ L'énoncé devient acceptable si un complément rétablit la borne:

5. *Il dormit depuis midi / Il dormit depuis le crépuscule jusqu’à l'aube.

Par contraste, l'imparfait cache systématiquement les bornes de l'événement qu'il décrit, comme l'atteste l'exemple 6, ce qui ne l'empêche cependant pas d'être utilisé dans des énoncés comme 7 où la vision des bornes est inévitable :4

\section{Il traversait la rue.}

2 Une unité linguistique conceptuelle comme \{chat $\}$ donne accès à un certain nombre d'informations dites « encyclopédiques », par exemple \{le chat a le poil doux $\}$ ou \{le chat aime boire du lait qui peuvent, au cas échéant, servir au destinataire à constituer un contexte d'interprétation de l'énoncé qui contient cette unité. Selon l'hypothèse présentée dans ces articles, l'imparfait, à la différence des autres temps du passé, impose l'usage de telles informations pour l'interprétation de l'énoncé. Ces articles sont François Heenen, «Imparfait et Stéréotypes », Milli mála 7/2015, Reykjavík : Institut Vigdís Finnbogadóttir, pp. 121-149 et François Heenen, «Imparfait et modalité », Milli mála 8/2016, Reykjavík : Institut Vigdís Finnbogadóttir, pp. 93-117 et également François Heenen, «L'imparfait, un temps à deux procédures », Milli mála 7/2017, Reykjavík : Institut Vigdís Finnbogadóttir, pp. 97-120.

3 Sur cette propriété aspectuelle du passé simple et d'autres liées à l'aspect global voir Danielle Leeman, «Le passé simple et son co-texte : examen de quelques distributions ", Langue française, Temps et co(n)texte, éd. Jacques Bres, 138/2003, pp. 20-34.

4 Cet usage particulier, appelé « imparfait narratif » a fait l'objet de très nombreuses études, parmi lesquelles Louis de Saussure et Bertrand Sthioul, «L'imparfait narratif : point de vue (et images du monde) ", Cabiers de praxématique 32/1999, pp. 167-188; et Jacques Bres, L'imparfait dit narratif, Paris : CNRS, 2005. 
7. «Monsieur Chabot retirait son pardessus qu'il accrochait à la porte. ${ }^{5}$

Une troisième particularité est que le passé simple autorise seulement deux possibilités pour l'agencement temporel d'événements dans un énoncé : concomitance ou séquentialité. On remarque dans l'exemple suivant que les deux possibilités sont aussi pertinentes l'une que l'autre :

8. Sylvie joua à la flûte et Jean (l'accompagna/ joua ensuite) au piano.

Le recouvrement temporel partiel de deux événements, courant avec l'imparfait, est par contre impossible avec le passé simple comme le montre la comparaison de ces deux exemples :

9. Il traversa le pont quand le soleil se couchait.

10. Il traversa le pont quand le soleil se coucha.

Il y a encore d'autres types d'oppositions entre le passé simple et l'imparfait qui ont été relevées par les études précédentes. Sur le plan discursif, on a constaté que le passé simple était particulièrement courant dans des contextes où on s'attend à ce que les événements se suivent temporellement. Donc le passé simple, plus que tous les autres temps du passé, favorise l'ordre positif alors que l'imparfait favorise plutôt l'ordre zéro : 6

11. Pierre entra. Marie téléphona.

12. Pierre entra. Marie téléphonait.

Cela expliquerait pourquoi dans l'exemple suivant l'ordre négatif est impossible ;

5 L'exemple vient de Georges Simenon, La danseuse du Gai-Moulin. Il est cité originellement dans Liliane Tasmowski-De Ryck, « L'imparfait avec et sans rupture », Langue française, 67/1985, éd. n.c., lieu d'éd. n.c., pp. 59-77, ici p. 75.

6 Voir l'analyse discursive des temps dans Hans Kamp et Christian Rohrer, "Tense in texts ", Meaning, Use, and Interpretation of Language, 1983, éd. Bauerle R., Schwarze C. et von Stechow A., Berlin et New York, de Gruyter, pp. 250-269. 
13. Les passagers descendirent. Lavion *atterrit à $20 \mathrm{~h}$.

Mais il y a beaucoup d'exceptions à cette tendance du passé simple. En effet l'interdiction de l'ordre négatif peut être contrecarrée par la présence d'un connecteur temporel dans l'énoncé.

14. Les passagers descendirent dès que l'avion atterrit.

Lordre zéro est lui évident dans les séquences d'énoncés dits encapsulants et encapsulés :?

15. «Une terrible tempête fit rage. Un arbre fut arraché. ${ }^{8}$

L'imparfait peut quant à lui permettre l'ordre positif notamment à travers l'usage stylistique narratif :

16. «Toujours courant, elle se débarrassait de sa veste, l'accrochait au passage à une patère du vestibule, sautait d'un bond dans le jardin en appelant : « Hé ! Ho ! »

Encore sur le plan discursif on dit que le passé simple communique des événements nouveaux qui servent à construire ou faire avancer la trame d'un récit narratif alors que l'imparfait en général analyse des faits déjà communiqués ou se limite à donner des informations d'arrière-plan $:^{10}$

17. Narcisse se promenait dans la forêt lorsqu'un bruit attira son attention.

7 L'encapsulation est le procédé qui consiste à expliciter des sous-événements d'un événement pour enrichir sa représentation.

8 Exemple cité dans Louis de Saussure, "Quand le temps ne progresse pas avec le passé simple », Cabiers Chronos 6/2000, pp. 37-48, ici p. 37.

$9 \quad$ Extrait de Pierre Bost, Monsieur Ladmiral va bientôt mourir, p. 17.

10 Au sujet de ces propriétés discursives voir Gilbert Dalgalian et Daniel Malbert, Grammaire textuelle du français, Paris : Didier/Hatier, 1989, p. 130, (traduction française de Harald Weinrich), Textgram-matik des französischen Sprache, Stuttgart : Klett, 1982. 
Mais là encore il y a de nombreux exemples qui contredisent cette tendance, dans lesquels l'imparfait communique un événement de premier plan :

18. « Le commandant [...] se jeta sur l'interphone et hurla qu'il avait à parler à $\mathrm{Mr}$ Chisnutt. Trois minutes plus tard, $\mathrm{Mr}$ Chisnutt se présentait chez le commandant. ${ }^{11}$

Une dernière propriété concerne uniquement l'imparfait, celle de nécessiter un ancrage temporel dans une autre situation. Cela expliquerait pourquoi un énoncé à l'imparfait isolé se laisse difficilement interpréter $:^{12}$

19. ? Il pleuvait.

20. Pierre rentra. Il pleuvait.

Comme annoncé au début de cette section, les propriétés de l'imparfait et du passé simple dont on parle dans la littérature sur ces deux temps sont multiples. Certaines sont constantes, d'autres admettent de nombreuses exceptions. Elles constituent le matériel de base dont se servent toutes les approches concernées par la fonction de ces deux temps, quels que soient leurs objectifs ou leurs bases théoriques.

\section{Passé et mémoire}

Une partie des énoncés formés avec les temps du passé servent à rapporter des souvenirs épisodiques de la vie du locuteur. Si par exemple je voulais vous raconter les vacances en Italie que j’ai passées avec ma femme et mes enfants en 2006 je le ferais surtout en utilisant des temps du passé : imparfait, passé composé et éventuellement plusque-parfait et conditionnel passé. Le début du récit serait quelque chose comme ceci :

11 Pour cet usage particulier dit « de rupture » voir Anne-Marie Berthonneau et Georges Kleiber, «Pour une réanalyse de l'imparfait de rupture dans le cadre de l'hypothèse anaphorique méronomique », Cabiers de praxématique 32/1999, pp. 119-166.

12 Cette propriété est à la base de l'approche anaphorique de l'imparfait, voir Anne-Marie Berthonneau et Georges Kleiber, « Pour une nouvelle approche de l'imparfait. L'imparfait, un temps anaphorique méronomique » Langages 112/1993, pp. 55-73. 
« Nous avons pris l'avion jusqu’à Amsterdam et de là le train pour Bruxelles où nous sommes restés deux jours. Ensuite nous avons continué jusqu'à Milan pour rendre visite à ma belle-sœur qui habite là. Je me souviens que nous sommes arrivés chez elle le soir et nous avons mangé dans un restaurant juste à côté. Il y avait des moustiques énormes qui n'arrêtaient pas de nous piquer. »

Pourquoi ai-je choisi l'imparfait pour parler des piqûres de moustiques ? Probablement parce que je ne me suis souvenu d'aucun moment précis pour cette action. J'aurais pu dire, par exemple « $\mathrm{Ma}$ femme s'est fait piquer par un moustique quand elle mangeait son dessert ", mais je n'ai pas de tel souvenir de cet épisode et il ne me serait pas venu à l'esprit de l'inventer juste pour les besoins du récit. Comment me suis-je souvenu des moustiques ? Probablement en me rappelant le souvenir du dîner au restaurant. Mais j'en ai parlé aussi parce que je savais que les moustiques allaient nous rendre la vie difficile durant tout notre séjour à Milan. Une autre influence du souvenir des piqûres de moustiques est qu'il m'a rappelé que tous les adultes à table ce soir-là, moi y compris, nous avons fumé au moins une cigarette chacun, pensant que c'était un moyen d'éloigner les insectes.

Un autre élément que j’aurais pu exprimer dans ce récit, bien que je ne l'aie pas fait, aurait illustré l'usage de l'imparfait. Je ne me suis pas souvenu clairement de ce que nous avions fait à Bruxelles les deux premiers jours, mais une image m'est tout de même venue à l'esprit, celle de nous tous dans un magasin $\mathrm{H} \& \mathrm{M}$ du centre-ville et particulièrement d'une de mes filles allant de sa propre initiative essayer des vêtements dans une cabine d'essayage, ce qui pour son âge à l'époque était un comportement assez inattendu. Je dirais que cette image m'est venue spontanément à l'esprit. Elle est connectée à mes souvenirs de vacances en famille mais je ne suis pas sûr qu'elle corresponde temporellement à celles de 2006 et c'est la raison pour laquelle elle est restée tacite.

Parlons maintenant des énoncés au passé composé dans ce court récit. Ils correspondent à des tâches précises que j'ai imposées à ma mémoire. Une de ces tâches était de me rappeler suffisamment d'événements pour que le récit ait un quelconque intérêt pour un auditeur 
imaginaire. Une autre tâche était de faire un récit cohérent en présentant les événements dans un ordre chronologique. Je ne me suis pas forcément souvenu des épisodes dans le même ordre que celui indiqué par les phrases du récit. En fait, mes souvenirs de vacances de 2006 concernent surtout les 15 jours que nous avons passés au bord de mer après le passage à Milan. Mais j’ai fait l'effort de me souvenir d'événements qui ont précédé ce séjour balnéaire parce que j’ai senti que c'était une façon plus logique de commencer la narration.

Ce court récit nous indique que l'usage des temps du passé peut réellement dépendre du travail de la mémoire effectué par le locuteur. Les exemples à l'imparfait illustrent des cas de souvenirs qui viennent spontanément à l'esprit, soit à travers un autre souvenir soit à travers le thème général qui guide la mémoire, alors que ceux au passé composé sont des souvenirs récupérés avec une tâche préalablement fixée. En quoi ces observations nous aideraient-elles à comprendre les propriétés aspectuelles des temps du passé ? Pour répondre à cette question, il est nécessaire de poser une hypothèse sur la fonction des temps du passé inspirée par la théorie modulaire de l'esprit et par son application en sémantique procédurale.

\section{Modularité massive et procédures}

La théorie modulaire voit le système cognitif de l'être humain comme constitué de modules biologiques, spécialisés dans le traitement de certains types de représentations. ${ }^{13}$ Le traitement consiste à appliquer sur les représentations des procédures spécifiques pour, soit les améliorer, soit les éliminer, ou pour produire à partir d'elles de nouvelles représentations. Les modules peuvent former des réseaux, ou systèmes, ce qui leur permet de communiquer entre eux le résultat de leur activité inférentielle. Certains modules sont innés, d'autres acquis par l'expérience. Deux particularités importantes caractérisent la théorie modulaire. La première est de concevoir que le système cogni-

13 Cette présentation de la théorie modulaire de l'esprit est basée sur Hugo Mercier et Dan Sperber, The Enigma of Reason, 2017, Harvard University Press. Le terme " représentation » dans le cadre de cette théorie désigne tout objet matériel doté d'une fonction cognitive, que ce soit par exemple un message écrit, un signal électrique, ou une représentation mentale interne, c'est-à-dire un réseau de neurones activés. 
tif utilise des méthodes d'inférence différentes selon le type de représentation qu'il traite. La seconde est de considérer que ces méthodes d'inférence sont en général inconscientes, sauf si elles sont métareprésentées par un module particulier. En s'appuyant sur la théorie modulaire, des auteurs ont suggéré que les unités du discours dites " procédurales » servaient à communiquer l'activité de modules déterminés. ${ }^{14}$ Donc, une conjonction comme \{mais\}, par exemple, au lieu de communiquer directement des procédures fixes que l'interlocuteur appliquerait sur l'énoncé, comme l'avaient suggéré d'autres auteurs auparavant, encouragerait l'interlocuteur à interpréter l'énoncé à travers un module. Cette approche a l'avantage d'expliquer pourquoi le sens des unités procédurales est si difficile à définir. La raison est que la méthode d'inférence utilisée par le module pour traiter l'énoncé échappe le plus souvent à la conscience de l'interlocuteur. Pourtant les procédures représentent la régularité du traitement quapplique le module sur l'énoncé. Elles doivent donc avoir une réalité psychologique mais la question est de comprendre le processus qui permet leur inférence. L'explication serait que l'interlocuteur qui traite un énoncé incluant une unité procédurale comme \{mais\} peut, en plus d'inférer à travers le module la nouvelle forme élaborée de l'énoncé, se représenter également les raisons pour lesquelles il a obtenu cette forme. Cette activité métareprésentative et argumentative sur les raisons de son propre travail inférentiel peut lui procurer des effets cognitifs supplémentaires et engendrer de cette manière un usage régulier des mêmes procédures. En d'autres mots, il peut métareprésenter les raisons qui l'ont poussé à obtenir la bonne nouvelle forme de l'énoncé.

14 Le terme " procédural » était au départ réservé aux connecteurs discursifs tels que, en anglais, « but », « so » ou « after all », qui n'encodent pas de concepts. La notion s'est vue ensuite appliquée à des expressions non syntaxiques comme " please ", « alas » ainsi qu’à des unités non-linguistiques telles que les expressions du visage ou la tonalité de la voix. Pour une synthèse sur l'évolution de la sémantique procédurale voir Robyn Carston, "The heterogeneity of procedural meaning », Lingua 175-176/2016, pp. 154-166. Pour l'analyse procédurale des morphèmes temporels et aspectuels du verbe voir Louis de Saussure, Temps et pertinence, 2003, De Boeck/Duculot, Bruxelles ; et Jacques Moeschler, Cristina Grisot et Bruno Cartoni, « Jusqu'où les temps verbaux sont-ils procéduraux ? ", Nouveaux cabiers de linguistique française, 30/2012, pp. 119-139. L'approche modulaire de la sémantique procédurale est défendue notamment dans Deirdre Wilson, "The conceptual-Procedural Distinction : Past, Present and Future », Procedural Meaning : Problems and Perspectives, Current Research in Semantics / Pragmatics Interface 25/2011, éd. Victoria Escandell-Vidal, Manuell Leonetti et Aoife Ahern, pp. 3-31. 
En nous basant sur cette conception du terme « procédure », imaginons qu'il existe un module au service de la communication ostensive qui sactive lorsqu'il traite des énoncés dont la propriété est de représenter des souvenirs d'événements passés. Ce module déterminerait si l'activité mnésique qui est à l'origine du souvenir a suivi une tâche définie (= mémoire à tâche positive) ou si elle était spontanée (= mémoire spontanée). Dans un premier temps, nous allons considérer ces deux sous-propriétés comme intrinsèquement liées aux énoncés mais nous verrons dans la section 7 qu'il y a probablement un lien entre d'une part la mémoire à tâche positive et la mémoire épisodique, qui est déclarative, et d'autre part entre la mémoire spontanée et le réseau cérébral par défaut. Le passé simple, le passé composé et le plus-que-parfait seraient tous liés au travail de la mémoire à tâche positive, l'imparfait seul marquerait la mémoire spontanée. Nous allons voir dans les deux sections suivantes que les énoncés qui représentent un souvenir d'événement passé sont construits de manière à mettre en évidence le type d'activité mnésique indiqué par le morphème grammatical. Inversement, ceux qui sont incompatibles avec le temps du verbe le sont parce qu'ils ne mettent pas en évidence le bon type d'activité mnésique. En d'autres termes, le module, qui n’a évidemment pas accès à l'historique de la mémoire, a la capacité de reconnaître dans l'énoncé même les propriétés qui confirment soit que la mémoire a travaillé en suivant une tâche fixe, soit qu'elle a travaillé sans suivre de tâche.

\section{Le passé simple}

Reprenons l'exemple 13 et essayons de comprendre pourquoi il ne correspond pas à l'idée qu’on se fait du travail de la mémoire à tâche positive :

13b) Les passagers descendirent. L'avion *atterrit à $20 \mathrm{~h}$.

Ce qui dérange dans cet exemple c'est qu'on se demande quel motif a incité la mémoire du locuteur à récupérer le souvenir de l'atterrissage, puisqu'en principe celui des passagers sortant de l'avion suppose la certitude que l'avion a atterri. En d'autres mots : pourquoi a-t-il 
besoin de se rappeler l'atterrissage s'il est capable de se rappeler le débarquement?

Avant de continuer cette analyse, posons deux conditions que devrait respecter tout énoncé au passé simple. Si l'on accepte l'hypothèse que ce temps représente le fonctionnement de la mémoire à tâche positive, on devrait s'attendre à ce que chaque énoncé mette en évidence deux caractéristiques mentales du locuteur :

- Avoir en tête une motivation interne pour entamer une recherche dans la mémoire.

- Ressentir un effet cognitif en récupérant le souvenir visé par la tâche positive.

Le deuxième énoncé de l'exemple 13b semble mal formé parce qu'il ne respecte aucune de ces deux conditions. Il ne met en évidence ni d'effet cognitif que ressentirait le locuteur, ni la motivation de ce dernier pour la récupération du souvenir. La seule possibilité serait d'imaginer que le locuteur se soit subitement demandé à quelle heure l'avion avait atterri. Mais cette question semblerait hors de propos vis-à-vis de la première phrase. ${ }^{15}$

Parmi les exemples soi-disant erronés qu'utilisent les auteurs pour démontrer les propriétés aspectuelles du passé simple, nombreux sont ceux qu'on pourrait expliquer de la même manière que l'exemple 13b.

10b) Il traversa le pont quand le soleil se coucha (couchait).

La raison pour laquelle cet énoncé ne peut pas communiquer le même sens que sa variante avec l'imparfait est que si le coucher du soleil était une circonstance de la traversée du pont, comme ce serait le cas avec l'imparfait, son souvenir aurait dû venir à l'esprit du locuteur au moment où il s'est rappelé celui de la traversée. Donc \{le soleil se coucha\}, s'il représentait cette circonstance, ne semblerait pas rapporter d'image mentale nouvelle pour le locuteur, pas plus que celui de l'atterrissage après le souvenir du débarquement dans $13 \mathrm{~b}$.

15 Le fait que le plus-que-parfait est par contre acceptable dans cet exemple (L'avion avait atterri à 20b) est discuté ici dans cet article pp. 106-110, dans la section consacrée au plus-que-parfait. 
La différence entre les énoncés de la catégorie « énoncés encapsulés » et ceux que nous venons de voir tient dans le fait que ceuxlà mettent clairement en évidence la motivation d'analyser en détail un autre souvenir, celui qualifié d'encapsulateur :

21. Ce samedi marqua le début de la relation de Paul et de Mane. Ils déjeunèrent ensemble. Ils se promenèrent sur les berges. Le soir, ils s'embrassèrent pour la première fois.

Ici les souvenirs reviennent petit à petit à la mémoire du locuteur. D'abord celui assez vague que quelque chose durant la journée du samedi a marqué le début de la relation et ensuite ceux des événements précis qui ont constitué cette journée. On ressent les effets cognitifs du locuteur en lisant chaque énoncé, et on comprend la motivation qui l'a guidé. Ce qui crée le sentiment d'incohérence de la mémoire dans les exemples $13 \mathrm{~b}$ et $10 \mathrm{~b}$ qu'on ne retrouve pas dans 21 , c'est le fait que les énoncés semblent reprendre une composante du premier souvenir, celle de l'atterrissage de l'avion, ou celle du coucher de soleil, ce qui procure un sentiment de redondance, complètement opposé à celui d'un effet cognitif. Ce sentiment de redondance n'émane pas de l'énoncé 21.

Les exemples ci-dessous montrent que la valeur aspectuelle du verbe s'adapte avec le reste de l'énoncé pour produire l'interprétation d'une situation nouvelle dont le souvenir procure un effet cognitif au locuteur :

22. ?Le trottoir fut mouillé après la pluie.

23. Le trottoir fut mouillé encore deux heures après la pluie.

24. Jean fut malade encore longtemps après son traitement. ${ }^{16}$

Pourquoi \{être mouillé\} reçoit-il une fausse valeur inchoative, voire passive dans 22 alors que les verbes dans 23 et 24 gardent leur aspect statif ? Lexplication serait que les situations décrites dans ces deux derniers exemples, grâce à l'adverbe encore, sont vues comme nouvelles, ce qui est propice au signalement d'un effet cognitif du locu-

16 Cet exemple et le précédent ont été suggérés par un relecteur anonyme. 
teur. On remarque d'ailleurs que pour la même raison, l'exemple suivant est également acceptable :

25. Le trottoir resta mouillé après la pluie.

Dans l'exemple 22, si \{fut mouillé\} était statif, l'énoncé serait interprété comme reprenant une circonstance de l'événement de la pluie, événement qui, étant donné l'article défini $\{$ la\} semble déjà avoir été récupéré par le locuteur. C'est pour cette raison que lorsqu'on interprète cet énoncé on modifie inconsciemment l'aspect du verbe pour créer artificiellement l'effet d'une nouvelle situation. Si \{pluie\} était indéfini, l'impression de redondance disparaitrait et le verbe reprendrait son sens statif :

26. Le trottoir fut mouillé après une pluie diluvienne.

L'impression d'incomplétude du souvenir peut également contredire l'image qu'on se fait du travail de la mémoire à tâche positive, d'où l'importance de la visibilité des bornes actionnelles de l'événement. Cela expliquerait l'incompatibilité avec \{depuis\} dans cet exemple :

5b) *Il dormit depuis midi.

Les études sur le passé simple ont relevé des propriétés multiples et disparates de ce temps telles que la tendance à imposer l'ordre positif, la nécessité de montrer les bornes de l'action, l'incompatibilité avec le recouvrement partiel d'événements, la fréquence de l'aspect inchoatif. Selon l'analyse faite dans cette section, ces propriétés n'ont d'autre but que de confirmer que l'énoncé rapporte un souvenir géré par la mémoire à tâche positive. Ces propriétés ont donc toutes un statut auxiliaire, ce qui explique leur inconstance relative. Aucune d'elle n’est censée délivrer un sens particulier.

Le module d'analyse dont nous avons supposé l'existence dans la section 4, va détecter dans l'énoncé l'indication sur le souvenir apportée par le passé simple et également les indications apportées par les autres composantes de l'énoncé. Ces indications secondaires de l'énon- 
cé sont que le souvenir est non redondant, autonome et complet, et que par conséquent il constitue un effet cognitif pour le locuteur. La conclusion du module sera que l'énoncé représente en lui-même l'effet cognitif que le locuteur a ressenti à travers le souvenir et qu'il a l'intention de rendre manifeste.

\section{L’imparfait}

L'objectif de cette section est de montrer que l'absence de tâche fixe dans l'activité mnésique, qui selon notre hypothèse caractérise la récupération des souvenirs que rapporte l'imparfait, explique les propriétés aspectuelles et discursives des énoncés à ce temps.

Dans le cas du passé simple, le fait de savoir que le locuteur s'est fixé une tâche pour la récupération implique que l'énoncé décrit le plus littéralement possible le souvenir qui lui procure des effets cognitifs. L'interlocuteur ne peut pas anticiper de cette manière les effets cognitifs de l'énoncé s'il sait que le souvenir est venu spontanément à la conscience du locuteur, parce que ce souvenir peut n'être qu'un fragment d'événement et que les effets cognitifs n'apparaîtront que postérieurement lorsque d'autres souvenirs viendront à la suite du premier. Cet effet retardataire des effets cognitifs explique pourquoi l'imparfait ralentit le flot informatif et comment il constitue souvent l'arrière-plan du discours. Larrière-plan est dû au fait que les souvenirs restent dans la mémoire du locuteur après leur communication parce que ce dernier a l'intuition qu'ils lui serviront encore par la suite. $^{17}$

La propriété anaphorique de l'imparfait s'explique par le fait que le souvenir vient souvent spontanément à partir d'un autre. C'est de cette manière que dans le bref récit de mes vacances de 2006 je me suis souvenu des moustiques en pensant au dîner au restaurant. Pour l'interlocuteur, identifier ce « souvenir-mère » peut aider à calculer les effets cognitifs de l'énoncé. Voyons un exemple de tel usage :

17 Voir à ce sujet Joseph P. Magliano et Michelle C. Schleich, «Verb Aspect and Situation Models », Discourse Processes 29(2)/2000, pp. 83-112 qui constate à travers des expérimentations qu'un événement reste plus longtemps en mémoire active lorsqu'il est exprimé avec le progressif plutôt que par une forme verbale perfective. 
27. «Peu après cette entrevue, je rencontrai Ivor Black, une seconde fois, à quelque réception au cours de laquelle il m'invita, en compagnie de cinq autres condisciples, à passer l'été dans une villa de la Côte d'Azur qu'il venait d'hériter, dit-il, d'une vieille tante. Il était très saoul, et il parut surpris lorsqu'à la veille de son départ, sept ou huit jours plus tard, je lui rappelai son exubérante invitation. $»^{18}$

Le pronom personnel \{il\} ainsi que le stéréotype que les gens au cours d'une réception boivent souvent beaucoup trop, nous montrent clairement que \{il était très saoul\} est un souvenir qui se rapporte à celui de la réception et de l'invitation formulée par Ivor Black. Nous déduisons de ce rapprochement qu'Ivor Black a invité le locuteur sous l'emprise de l'alcool et n'a donc pas eu pleinement conscience de ce qu'il disait. Cette implication doit constituer l'effet cognitif qu'a ressenti le locuteur en se représentant \{il était saoul\}.

Les énoncés à l'imparfait sont souvent formulés de telle façon qu'on les rattache aisément à une situation connue ou facilement inférable. C'est le cas très fréquent des phrases complexes dont un des verbes est à l'imparfait :

9b) Il traversa le pont quand le soleil se couchait.

Le souvenir du coucher du soleil modifie celui de la traversée du pont en y ajoutant la vision d'une luminosité et d'un paysage particuliers.

Les propriétés discursives et anaphoriques des énoncés à l'imparfait, dont nous avons parlé, ne sont pas constantes parce qu'un souvenir spontané ne doit pas nécessairement être diffus et généré par un autre souvenir. Il peut être parfaitement clair et surgir brusquement à la mémoire du locuteur. Les trois énoncés ci-dessous ne contredisent donc pas l'image qu'on se fait de la mémoire spontanée :

18b) « Le commandant [...] se jeta sur l'interphone] et hurla qu'il avait à parler à Mr Chisnutt. Trois minutes plus tard, Mr Chisnutt se présentait chez le commandant. »

18 Le passage provient de Vladimir Nabokov, Regarde, regarde les arlequins!', Traduit de l'anglais par Jean-Bernard Blandenier, 10-18, Série « Domaine étranger » dirigée par Jean-Claude Zylberstein, Fayard, 1974, p. 6. 
28. Elle ne voulut pas le revoir. Le lendemain, il partait pour la guerre.

29. Pierre alluma la lampe. La lumière donnait à la pièce un air de tristesse désolée.

Les procédures dont j’ai posé l'existence dans mes trois articles sur l'imparfait se justifient dans le cadre de la présente approche parce qu'elles facilitent l'interprétation pertinente des énoncés. ${ }^{19}$ Elles tiennent compte de la possibilité que le souvenir rapporté par l'énoncé puisse être diffus et basé sur un autre souvenir. L'application de ces procédures est responsable d'autres propriétés aspectuelles et temporelles de l'imparfait, notamment la concomitance avec le moment indiqué par un complément temporel, la vision médiane de l'événement et l'atélicité.

La première procédure impose de compléter la forme propositionnelle de l'énoncé par une référence temporelle. Celle-ci peut être le souvenir-mère si le cotexte le met suffisamment en évidence. La coréférence parfaite entre l'événement décrit par l'énoncé à l'imparfait et celui qui sert de référence s'explique donc par l'origine mnésique commune des énoncés qui les rapportent :

30. J'entrai dans la pièce. Jeanne écrivait une lettre.

Le souvenir de l'action télique d'écrire une lettre est issu de celui du locuteur entrant dans la pièce, d'où le recouvrement parfait des deux actions. ${ }^{20}$

Si le cotexte n'offre pas la possibilité d'inférer un souvenir-mère, la procédure encouragera l'interlocuteur à déterminer un complément temporel.

29b) « Pierre alluma la lampe. La lumière donnait à la pièce un air de tristesse désolée. »

19 Voir les références de ces articles ici dans cet article.

20 Un relecteur anonyme a fait pour cet exemple la remarque que l'action d'entrer était incluse dans celle d'écrire une lettre ce qui est évident si on prend en considération le début de cette dernière. On ne peut cependant nier qu'à la lecture de ce passage, les deux actions apparaissent comme concomitantes et aucun élément contextuel n'incite à se représenter le moment où Jeanne a commencé sa lettre. 
Ce complément pourrait être \{immédiatement après que la lampe ait été allumée\}, un moment auquel le locuteur aurait pensé et qui aurait provoqué le souvenir de l'air triste dans la pièce.

La deuxième procédure consiste à faire une implication, appelée RS (= représentation stéréotypée), à partir de la forme propositionnelle de l'énoncé, enrichie de la référence temporelle, et d'un ensemble d'hypothèses encyclopédiques attachées au concept encodé par le verbe. Reprenons l'exemple 30 :

30b) J'entrai dans la pièce. Jeanne écrivait une lettre.

$\mathrm{p}$ \{au moment où le locuteur entre dans la pièce Jeanne écrit une lettre\}

RS \{au moment où le locuteur entre dans la pièce Jeanne est assise à une table, un stylo en main, une feuille de papier devant elle, ...\}

La procédure tient compte de l'aspect potentiellement diffus du souvenir spontané dont nous avons parlé plus haut. L'énoncé peut être une synthèse d'images mentales que le locuteur entretient et qui constituent les fragments d'un événement. L'inférence des RS permet à l'interlocuteur d'avoir une vue plus précise de l'environnement cognitif du locuteur au moment où le souvenir spontané lui est venu à l'esprit. Ces images fragmentées de l'épisode sont importantes parce qu'elles peuvent générer d'autres souvenirs, comme dans la RS de l'exemple 30, où \{table\} peut rappeler au locuteur le souvenir d'un objet sur la table et $\{$ stylo\} la couleur du stylo.

Mais l'environnement cognitif dans certains exemples peut être beaucoup plus stable comme dans le cas de \{Il était saoul\} de l'exemple 27 où l'on sait grâce au cotexte quelles images stéréotypées de \{être saoul\} le locuteur a l'intention de communiquer.

La vue médiane de l'action et l'atélicité sont causées par l'inférence des RS et l'influence de la mémoire encyclopédique. Mais l'atélicité vient aussi du fait que les RS représentent des fragments d'un souvenir qui lui a une référence temporelle et une structure actionnelle fixes. Si le souvenir se rapporte à la phase finale d'une action, les fragments seront reliés à elle, s'il concerne le début, les fragments représenteront le début, mais les fragments d'un seul souvenir ne vont pas être reliés à différentes phases. Cela explique l'incompatibilité de 
l'imparfait avec des compléments temporels indiquant plusieurs phases.

31. ?Je faisais la vaisselle en dix minutes.

\section{Au sujet de la mémoire déclarative et du réseau cérébral par défaut}

La mémoire déclarative à long terme est définie comme la capacité de se remémorer consciemment des faits ou des événements dans le but de s'en faire une représentation mentale stable qu'on peut exprimer par le langage. ${ }^{21}$ Parmi les différents types de mémoire déclarative, il y a la mémoire épisodique que nous utilisons quotidiennement pour nous rappeler des événements que nous avons personnellement vécus comme par exemple l'heure d'un rendez-vous qui nous a été donné ou le cadeau que nous avons reçu pour notre dernier anniversaire. Le parallèle entre cette activité mnésique et les énoncés au passé simple est justifié dans la mesure où ces énoncés expriment les parties délimitées d'un récit, qui ont une référence temporelle et une structure actionnelle fixes. Les souvenirs qu'ils représentent ne peuvent qu'avoir été produits par une activité consciente du système cognitif. Généralement les mémoires déclaratives sont opposées aux non déclaratives qui, elles, sont implicites et inconscientes. Mais pour justifier le terme « mémoire spontanée » et son lien avec les énoncés à l'imparfait nous n'allons pas nous référer aux mémoires non déclaratives mais à une activité cognitive appelée le réseau cérébral par défaut. Le réseau cérébral par défaut (RD), qui comprend plusieurs groupes de régions de neurones, est plus actif lorsque le cerveau est au repos que lorsqu'il est occupé par une tâche dirigée vers un objectif spécifique, et inversement, il est moins actif lors de l'accomplissement de telles tâches. ${ }^{22}$ Une des hypothèses concernant sa fonction est qu'il sert à maintenir une activité neurologique minimale pendant les moments de repos

21 Voir Larry R. Squire, « Memory and Brain Systems : 1969 -2009 », The Journal of Neuroscience, October 14/2009, pp. 12711-12716; et LARRY R. Squire et Stuart M. Zola, « Structure and function of declarative and nondeclarative memory systems », Proc. Natl. Acad. Sci. USA, 93/1996, pp. 13515-13522.

22 Voir Christine Bastin, «Le réseau cérébral par défaut : un repos qui n'en est pas un », Revue Neuropsychologie 10 (3)/2018, pp. 232-238. 
pour éviter de devoir les redémarrer complètement au moment voulu lorsque le cerveau redevient actif. ${ }^{23}$ On a d'ailleurs constaté que les mêmes régions qui sont actives durant certaines tâches cognitives, notamment la mémoire épisodique et autobiographique, le sont également au sein du RD. Voici ce que nous dit Christine Bastin à ce sujet :

«Ainsi, les études en IRMf de la mémoire épisodique identifient le cortex cingulaire postérieur et le cortex pariétal inférieur comme les corrélats cérébraux du succès de la récupération d'une information en mémoire et souligne que ces régions sont superposables avec les parties postérieures du réseau par défaut [...]. Par ailleurs, lorsqu'un individu évoque un souvenir autobiographique de nature épisodique, le réseau cérébral qui sactive est pratiquement identique au réseau par défaut, incluant à la fois ses composantes postérieures (cortex cingulaire postérieur et cortex pariétal inférieur) et ses composantes antérieures (cortex préfrontal médial, hippocampe, cortex temporal latéral). $»^{24}$

Dans des expérimentations visant à déterminer le contenu mental du RD, des participants ont été interrogés sur les pensées qu'ils avaient eues durant une période de repos. Dans leurs réponses, ils disaient avoir eu des pensées autobiographiques, des images mentales liées à leur vie personnelle et des planifications d'événements futurs. La mémoire n'est donc pas la seule tâche cognitive active qui se reflète dans l'activité au repos du RD mais elle en fait clairement partie. Une autre indication du lien avec la mémoire est que les régions cérébrales les plus touchées par la maladie d'Alzheimer sont dans le réseau par défaut. Certains auteurs pensent même que le RD est directement impliqué dans le fonctionnement de la mémoire épisodique. Ainsi Daniel Hutto défend l'opinion que le RD procure des « simulations imaginaires » pouvant servir d'éléments de narration. ${ }^{25}$ Il rejoint ain-

23 Une autre hypothèse serait que le $\mathrm{RD}$ prépare l'individu aux événements à venir en intégrant dans la personne les expériences remémorées et en réalisant avec eux des scénarios potentiels.

24 Bastin : Le réseau cérébral, p. 235. Un grand nombre de publications traite du rôle du RD dans la mémoire épisodique, notamment Carlo Sestieri, Maurizio Corbetta, Gian Luca Romani et Goldon L. Shulman, «Episodic memory retrieval, parietal cortex, and the default mode network : functional and topographic analyses », 31/2011, pp. 4407-20.

25 Voir Daniel D. Hutto, « Memory and narrativity », 2017, https://www.academia.edu/25340514/ Memory_and_Narrativity, pp. 2-37, ici p.15-16, citant aux mêmes pages Philip Gerrans, The measure of madness, 2014, Cambridge, MA : MIT Press, p. 17. 
si une opinion bien ancrée dans le domaine des recherches scientifiques sur la mémoire que "se souvenir c'est imaginer le passé ».

La recherche sur le fonctionnement du RD est un domaine actif, mais beaucoup d'éléments restent à élucider et il faut éviter de généraliser des propriétés qu'on a accordées au RD sur base d'expérimentations à portée limitée.

Quel rapport y a-t-il entre les énoncés à l'imparfait et le RD ? Précisons d'abord que l'hypothèse faite dans cet article n'est pas d'affirmer que l'alternance des temps du passé, par exemple dans un récit, reproduit en direct l'activité de la mémoire déclarative et du RD, mais plutôt de montrer que le récit imite ou simule ces activités cognitives. Parmi les propriétés discursives de l'imparfait, nous retiendrons spécialement le fait qu'il interrompt des séquences d'événements exprimés avec le passé simple ou le passé composé, produit des effets cognitifs implicites qui souvent n'apparaissent quavec l'interprétation d'autres énoncés, forme des arrière-plans, ralentit le flot informatif. Le rapprochement entre ces propriétés et celles des souvenirs récupérés par le RD est évident. Le souvenir issu du RD marque une pause après le travail actif de la mémoire déclarative, il maintient néanmoins cette activité à un niveau moins élevé d'où les effets cognitifs diffus. Un autre caractère du RD que les énoncés à l'imparfait reproduisent, c'est de préparer la reprise de la mémoire déclarative en récupérant des souvenirs qui lui seront utiles.

\section{8. Ébauche d'une analyse du passé composé}

Les études sur le passé composé mettent en évidence un usage particulier dans lequel le verbe implique une autre situation que celle qu'il décrit, situation qui est vraie au moment de l'énonciation. ${ }^{26}$ Cet usage est considéré comme spécifique pour le passé composé et incommunicable par le passé simple. Voici un exemple :

32. Il a plu > \{le linge qui pend dehors est mouillé $\}>$ \{il faut le rentrer\}

26 Voir Jean-Marc Luscher et Bertrand Sthioul, «Emplois et interprétations du passé composé », Cahiers de linguistique française, 18/1996, pp. 187-217. 
Selon l'explication la plus courante pour cet usage, la situation vraie au présent serait inférable à partir d'un schéma encyclopédique, en l'occurrence ici : \{si il pleut sur un objet l'objet est mouillé\}.

Mais une autre explication pour le processus d'interprétation de tels énoncés est également possible et met en jeu la mémoire à tâche positive, permettant ainsi de dégager une propriété commune avec le passé simple. Supposons qu'en se posant la question \{faut-il ou ne fautil pas rentrer le linge\} le locuteur se soit rappelé l'événement passé de la pluie. S'il est mutuellement manifeste au locuteur et à l'interlocuteur que l'énoncé au passé composé représente un souvenir suscité par une tâche positive, l'interlocuteur va chercher à se représenter cette tâche. En cherchant parmi les hypothèses les plus accessibles, il va trouver la même question que s'est posée le locuteur. S'étant assuré que le souvenir de la pluie a procuré un effet cognitif au locuteur, l'interlocuteur considère que l'énoncé est pertinent en soi et peut être exploité comme une prémisse pour d'autres inférences. De cette manière, il aboutira à la conclusion \{il faut rentrer le linge\} comme l'avait prédit l'autre explication.

Sur le plan du travail de la mémoire, cet exemple ne prouve donc aucune différence entre le passé composé et le passé simple, raison pour laquelle ces deux temps sont utilisés dans les récits. Mais la différence entre eux peut résider dans un autre domaine. Comparons ces deux énoncés :

33. Jeanne est arrivée à l'heure au bureau. Elle s'est acheté un nouveau réveil.

34. Jeanne arriva à l'heure au bureau. Elle s'acheta un nouveau réveil.

L'exemple 33 est cohérent et peut s'expliquer de la même manière que l'exemple 32. Le souvenir que Jeanne s'est acheté un réveil a été suscité par la question \{mais comment a-t-elle fait pour arriver à l'heure\} que le locuteur a considérée comme mutuellement manifeste grâce à la référence à Jeanne dans le premier énoncé, si l'on imagine que les deux interlocuteurs connaissent les problèmes de ponctualité de Jeanne. Avec le passé simple, l'exemple 34 est par contre incohérent et est interprété comme une séquence décousue d'événements. L'expli- 
cation pour cette différence serait que le motif de la recherche de la mémoire, dans le cas du passé simple, n’est pas supposé être dans un environnement cognitif qui comprendrait l'interlocuteur. Il n'y a donc pas moyen de sélectionner une représentation mutuellement manifeste pour activer la mémoire. Le passé composé n’imposerait aucune restriction concernant le motif de la mémoire.

Une autre particularité du passé composé est que certains énoncés ne font aucune référence au travail de la mémoire :

35. Un client dans un restaurant s'adressant à un serveur : « J'ai fini. »

36. B à qui $\mathrm{A}$ explique une démonstration de mathématiques : «Maintenant, j’ai compris. ${ }^{27}$

Dans les situations qu'on imagine pour ces énoncés, il n'est pas mutuellement manifeste que le locuteur allait formuler un énoncé représentant un souvenir. Il est néanmoins évident que ce qui a incité le locuteur à utiliser un temps du passé c'est que l'information qu'il veut communiquer est basée sur un souvenir : dans 35, par exemple, le client sait quand il a fini son repas. Il veut que cette information soit interprétable pour l'interlocuteur, mais il ne veut pas que ce dernier considère qu'il avait l'intention de la communiquer.

\section{9. Ébauche d'une analyse du plus-que-parfait}

Dans cette section sur le plus-que-parfait nous allons nous contenter d'examiner le problème de la dualité entre les deux usages qu'on appelle plus-que-parfait d'antériorité et plus-que-parfait de l'accompli. Le but sera de montrer que l'approche mnésique proposée dans cet article permet de concevoir le second usage comme constant ce qui permet d'éviter une catégorisation sans critère défini. ${ }^{28}$

27 Les usages du passé composé avec un déictique indiquant le présent sont discutés dans Louis de Saussure, «Le passé composé en usage futur et la pertinence au présent ». https://www. researchgate.net/profile/Louis_De_Saussure/publication/259422604_Le_passe_compose_en_ usage_futur_et_la_pertinence_au_present/links/546f7c990cf2d67fc031160c/Le-passe-composeen-usage-futur-et-la-pertinence-au-present.pdf, pp. 1-18; ici pp. 86-90.

28 Cette généralisation de la notion d'état impliqué est également proposée dans Saussure : Temps et pertinence, p. 249. 
D'après les analyses courantes du plus-que-parfait, ce temps indique soit un rapport d'antériorité entre deux actions dans le passé (= plus-que-parfait d'antériorité), soit un état résultant d'une action du passé (= plus-que-parfait de l'accompli). Izumi Tahara interprète ainsi l'exemple 37 comme une illustration du premier usage et 38 comme un exemple du second. ${ }^{29}$

37. « J'ai vu Claire à Noël. Elle avait de nouveau commencé à suivre un régime. Mais maintenant elle mange de nouveau comme avant. C'est dommage. »

38. « Hier je n’ai pas apporté son gâteau préféré chez Marie. Elle avait de nouveau commencé à suivre un régime. »

Dans 37, les trois énoncés représentent clairement trois situations sur la ligne du temps : \{elle avait de nouveau commencé à suivre un régime\} qui précède \{j’ai vu Claire à Noël\} qui elle-même précède \{maintenant elle mange de nouveau comme avant\}. Par contre dans 38, \{elle avait de nouveau commencé à suivre un régime\} décrit un état qui caractérise la situation du premier énoncé. Pourtant rien n'empêche dans 37 d'imaginer aussi un état, en l'occurrence que Claire était dans l'état de suivre un régime à Noël. Choisir l'une ou l'autre interprétation pour chacun de ces exemples semble plutôt relever d'un problème de pertinence. Le schéma temporel est bien marqué dans 37 et s'impose facilement à l'interlocuteur, alors que dans 38 la représentation de l'état aide manifestement à comprendre le premier énoncé.

Il semble évident qu’un énoncé au plus-que-parfait laisse toujours entrevoir une situation qui a lieu juste après celle décrite par l'énoncé. Ceci se constate même dans les exemples où le verbe au plus-queparfait exprime un état :

39. «Sa femme avait été folle de lui [...]; elle l'avait aimé avec mille servilités qui l'avaient détaché d'elle encore davantage. » ${ }^{30}$

29 Voir Izumi Tahara, Usage descriptif et usage interprétatif des temps du passé et des adverbes temporels dans le discours de fiction, Thèse de doctorat : Univ. Genève, 2004, DOI : 10.13097/archive-ouverte/ unige :574, p. 233.

30 L'extrait est cité et analysé dans Thara : Usage descriptif, p. 243. L'adverbe « autrefois » a volontairement été retiré de l'extrait pour qu'on puisse juger de la temporalité de l'événement sous-entendu sans sa contribution. 
Dans cet extrait de Madame Bovary, on sent bien qu'en communiquant cet énoncé, l'auteur pense déjà à une situation différente où les événements qu'il exprime avec le plus-que-parfait ne sont plus vrais. En comparaison, le passé simple ne sous-entend rien, la situation est uniquement celle décrite par l'énoncé $:{ }^{31}$

40. « elle l'avait aimé avec mille servilités »

41. « elle l'aima avec mille servilités »

Mais dans l'exemple 39, pour identifier cette situation sous-entendue, l'interlocuteur ne peut pas se baser sur un schéma encyclopédique, comme il le ferait dans cet autre exemple, dans lequel à partir de \{neiger\} on implique aisément \{être couvert de neige\}:

42. " Ce matin-là, il avait beaucoup neigé. Les enfants étaient contents de voir plein de neige à leur réveil. » ${ }^{32}$

Donc ce qu'on appelle l'état résultant est en fait une situation à laquelle pense le locuteur, mais que l'interlocuteur n'est pas forcément capable de définir. C'est de lui que dépend le choix d'utiliser des données encyclopédiques pour faire cette définition, si celles-ci sont suffisamment évidentes et si le résultat de l'inférence lui procure des effets cognitifs. On en revient donc à la remarque faite plus haut en considération des exemples 37 et 38, que la décision de l'interlocuteur de tenir compte ou pas de la situation sous-entendue par l'énoncé au plus-que-parfait dépend de la pertinence qu'il entrevoit dans cette inférence. Cette inférence n'est pas communiquée par le plus-que-parfait. Ce que ce temps communiquerait par contre, ce serait que le locuteur se représente cette situation à partir d'une autre situation. Dans le cadre de l'approche mnésique cela signifierait qu'il récupère

31 Ceci expliquerait l'usage du plus-que-parfait dans l'exemple 13b ici dans cet article p. 94. Un relecteur anonyme m'a demandé d'expliquer pourquoi on pourrait dire « les passagers descendirent. L'avion avait atterri à $20 \mathrm{~h}$ » alors que « Les passagers descendirent. L'avion *atterrit à $20 \mathrm{~h}$ » semble mal formé. La raison est que le passé simple décrit le souvenir de l'atterrissage, qui comme j'ai affirmé dans l'analyse de cet exemple est supposé déjà être clair à l'esprit du locuteur quand il récupère celui du débarquement des passagers. Mais le plus-que-parfait sous-entend une situation qui fait suite à l'atterrissage. Cette situation peut très bien susciter un intérêt particulier du locuteur, mais le manque de contexte ne permet pas de savoir en quoi il consiste.

32 Voir l'analyse de cet exemple dans Tahara : Usage descriptif, p. 239.

108 Milli mála 12/2020

10.33112/millimala.12.3 
un épisode, de la même manière qu'il ferait avec la mémoire à tâche positive représentée par le passé simple, et à partir de là imagine une autre situation dans le passé. Plusieurs raisons expliqueraient ce mode de travail de la mémoire. L'une serait qu'au moment où il récupère l'épisode qu'il exprime avec le plus-que-parfait, le locuteur n'a qu'un souvenir flou de la situation sous-entendue. La seule chose dont il serait certain c'est qu'une autre situation lui a fait place. Cette explication vaudrait pour l'exemple 39. Une autre raison serait que le locuteur n'ait pas le souvenir de la situation sous-entendue et qu'il la reconstruit à partir de l'épisode récupéré, en s'aidant d'un schéma d'actions. Pour illustrer une telle situation, considérons l'exemple suivant :

43. « Il fallut aller cueillir le grand oiseau d'or au milieu d'un champ de magnolias où ils'était doucement posé. »33

On peut supposer ici que le locuteur a le souvenir de l'oiseau se posant au milieu du champ de magnolias et également celui des personnes qui décident d'aller le cueillir. Mais il n'aurait pas celui de l'oiseau dans le champ. Pour se représenter cette situation, il se fierait au schéma $\{\{x$ se pose sur $y\}$ implique $\{x$ est sur y\}\}. Cet exemple montre également comment se produit le rapport d'antériorité entre l'événement décrit au plus-que-parfait et celui au passé simple. Puisque l'événement décrit au plus-que-parfait a été récupéré par la mémoire à tâche positive, il correspond à une étape franchie par la mémoire. Donc la situation sous-entendue ne peut que concerner un autre épisode avec une référence temporelle différente. Cela explique qu'un verbe au plus-que-parfait exprime toujours un rapport d'antériorité même s'il n'y a pas d'énoncé au passé simple à proximité. Si maintenant le cotexte rend compte d'un autre événement, comme celui de la prise de décision d'aller cueillir l'oiseau dans l'exemple 43, on suppose que la situation sous-entendue, l'oiseau au milieu du champ, est encore une circonstance de cet événement-là, qui ne peut donc être que postérieur à celui exprimé au plus-que-parfait.

33 Exemple cité dans Denis Apothéloz et Bernard Combettes, « Saillance et aspect verbal : le cas du plus-que-parfait », saillance. Aspects linguistiques et communicatifs de la mise en évidence dans un texte, 1/2011, Besançon: Presses universitaires de Franche-Comté, pp. 225-246, ici p. 230. 


\section{Le système des temps du passé du français selon l'hypothèse mnésique}

L'hypothèse que les temps grammaticaux du passé expriment différents modes de travail de la mémoire n'est pas justifiable uniquement à travers des analyses d'énoncés, qu'on pourra toujours considérer comme partiales. Dans les paragraphes qui vont suivre, nous allons tenter de montrer que le besoin de marquer spécifiquement différents modes de travail de la mémoire provient naturellement du discours narratif.

Imaginons un locuteur sur le point de faire un récit narratif et utilisant une forme grammaticale particulière, disons le passé simple, pour indiquer que pendant la communication du récit, sa mémoire épisodique sera active. L'interlocuteur a donc la présomption que les énoncés qu'il va interpréter représenteront chacun des produits de la mémoire épisodique. Il cherche à confirmer cette présomption. Donc, se basant sur sa propre connaissance du fonctionnement de la mémoire épisodique, il s'attend à ce que les énoncés représentent des souvenirs d'événements du passé qui ont été recherchés par la mémoire avec un motif décidé à l'avance, et dont la récupération procure au locuteur un certain effet cognitif. Pour reconnaître ces propriétés des souvenirs épisodiques à travers les énoncés, l'interlocuteur va se constituer des critères. L'un d'eux sera par exemple qu'un énoncé décrivant une action inchoative sera pertinent parce qu'il est aisé d'imaginer que le souvenir du début d'une nouvelle action procure un effet cognitif. De même, il est plus facile d'imaginer qu'un événement global ait pu constituer le motif d'une recherche mnésique, plutôt qu'un événement dont le déroulement est lié à celui d'un autre. Un troisième critère serait que l'énoncé décrive un événement temporellement subséquent à celui décrit par l'énoncé précédent puisque la suite dans le temps est un repère naturel dont se sert la mémoire épisodique pour inscrire deux événements.

Supposons que durant la communication du récit le locuteur récupère des souvenirs qu'il considère pertinents, mais qui ont des propriétés différentes des autres. Pour rapporter ces souvenirs, il a deux options : soit il utilise la même forme linguistique, le passé simple, et fait confiance à la perspicacité du destinataire pour sentir qu'il y a une 
différence entre ces souvenirs-là et les autres, soit il signale formellement que ces souvenirs sont différents en choisissant des formes spécifiques. S'il a l'intuition que ces souvenirs différents ont des propriétés régulières et qu’on peut les répartir en catégories, il va choisir la deuxième option. Supposons qu'il marque ces catégories respectivement avec l'imparfait et le plus-que-parfait.

Pour interpréter les énoncés à l'imparfait, l'interlocuteur remarquera que l'événement auquel le verbe fait référence a la plupart du temps des propriétés aspectuelles différentes de celles auxquelles font référence les énoncés au passé simple. En particulier les événements à l'imparfait sont des états, ou des activités sans bornes temporelles définies, qui sont difficilement concevables comme des cibles de la mémoire épisodique. Cette propriété des énoncés à l'imparfait n’est pas constante, mais l'interlocuteur la considérera comme l'indication qu'il faut associer ces énoncés avec un mode de travail mnésique dans lequel aucune cible prédéfinie ne joue un rôle déterminant. Par ce biais, il est donc facilement concevable que l'interlocuteur en arrive à la conclusion que les énoncés à l'imparfait décrivent des souvenirs qui viennent spontanément à l'esprit. Se fiant maintenant sur sa propre expérience de la mémoire spontanée des événements du passé, il sait que celle-ci a deux propriétés qu'il s'attend à pouvoir inférer à travers le co(n)texte : la première est d'être souvent suscitée par un souvenir épisodique précédemment récupéré, la seconde est de favoriser la récupération d'autres souvenirs, qu'ils soient eux également spontanés ou épisodiques. En conséquence, il applique à l'énoncé deux procédures qui confirment ces deux propriétés. La première consiste à déterminer un événement, explicitement ou implicitement indiqué par le contexte, que l'on pourrait associer au souvenir responsable du fonctionnement de la mémoire spontanée. Le fait que l'énoncé à l'imparfait inclut des éléments en coréférence avec des éléments d'autres énoncés à proximité constitue un indice pour l'interlocuteur que cette procédure est bel et bien intentionnée par le locuteur. À travers la deuxième procédure, l'interlocuteur se sert d'hypothèses encyclopédiques sur l'action ou l'état décrit par le verbe pour inférer des images mentales qui pourraient correspondre à celles que se ferait le locuteur à la suite du souvenir spontané. Ici également, l'interlocuteur s'attend à ce que cette procédure soit facilitée par le choix du verbe à l'impar- 
fait. Ce serait une autre raison pour laquelle certains types d'événements seraient moins courants à l'imparfait, comme des événements ponctuels ou des événements qui chevauchent différentes phases actionnelles.

Pour les énoncés au plus-que-parfait, l'interlocuteur constatera qu'ils correspondent à plusieurs critères utilisés pour les énoncés au passé simple. Il en conclut que ces énoncés décrivent des souvenirs épisodiques et non spontanés comme ceux à l'imparfait, et ce malgré la ressemblance morphologique entre ces deux catégories. Le point commun avec l'imparfait peut cependant résider dans le fait que le souvenir décrit par l'énoncé favorise la récupération d'un autre souvenir. Seulement comme le souvenir rapporté avec le plus-que-parfait a été récupéré selon une cible définie et est pertinent en soi, l'interlocuteur pensera que l'autre souvenir, qui est sous-entendu par le locuteur, doit avoir une référence temporelle différente. Pour identifier ce souvenir-là, l'interlocuteur ne va pas utiliser d'hypothèses encyclopédiques avec lesquelles il ne pourrait que compléter le souvenir épisodique, mais un schéma d'actions. Si aucun schéma d'actions n’apporte de résultat satisfaisant, l'interlocuteur peut tenter d'inférer le souvenir sous-entendu uniquement en se basant sur des éléments du contexte ou il peut tout simplement abandonner l'opération.

Dans cette fiction de la genèse du système des temps du passé du français nous avons vu que pour comprendre le lien entre le temps grammatical et un mode déterminé de travail de la mémoire, l'interlocuteur vérifie certains critères dans l'énoncé qui selon lui indiqueraient comment le souvenir a été récupéré. Un critère serait par exemple de voir si l'énoncé décrit un événement dans sa globalité, ce qui indiquerait que le souvenir vient de la mémoire épisodique, un autre consisterait à vérifier dans le cotexte s'il y a un événement coréférent, ce qui l'orienterait vers la conclusion que le souvenir est spontané. Le locuteur a tout intérêt à construire ses énoncés de telle manière à faciliter l'usage de ces critères puisque de cette manière il certifie l'interlocuteur que l'usage du temps grammatical est cohérent par rapport au mode de travail de la mémoire reflété par l'énoncé. Le respect de ces critères est donc utile pour maintenir un climat de confiance entre le locuteur et l'interlocuteur. On peut cependant imaginer des situations de communication dans lesquelles le locuteur 
formule un énoncé sans tenir compte de ces critères, bien qu'il soit réellement en train de récupérer un souvenir de la manière indiquée par le temps grammatical. Un souvenir spontané peut ainsi surgir de rien, un souvenir épisodique peut concerner un événement qui précède et non fait suite à celui décrit par l'énoncé précédent. Le non-respect des critères ne signifie pas que l'énoncé soit mal formé.

Deux caractéristiques essentielles de l'usage des temps du passé en français doivent encore être intégrées dans le modèle que nous avons proposé dans cette section :

- Le fait que les temps du passé sont utilisés dans des énoncés qui ne représentent pas des souvenirs épisodiques.

- La fonction du passé composé.

Commençons par le premier problème. L'hypothèse que le passé simple indique l'activité de la mémoire épisodique, c'est-à-dire la récupération consciente de souvenirs personnels d'événements du passé, semble à première vue contredire le fait que ce temps ne sert jamais en français moderne à communiquer des souvenirs épisodiques et que, même à l'époque où il avait un usage plus courant, on s'en servait aussi pour relater des événements dont on n’avait aucune expérience personnelle. Pour lever cette contradiction, nous devons faire l'hypothèse que le passé simple et les autres temps du passé peuvent faire référence au fonctionnement de la mémoire épisodique sans pour autant impliquer que ce fonctionnement soit réel au moment de la communication. Cette hypothèse repose sur le fait que nous sommes capables de nous représenter et de communiquer le fonctionnement de la mémoire épisodique. Par exemple, si au cours d'une conversation, je cherche à me rappeler ce que j'ai fait le week-end dernier, je peux signaler à mon interlocuteur que je suis en train de chercher ce souvenir, que j'ai trouvé des indices qui me mettent sur sa voie et finalement qu'il m'est venu à l'esprit. Si je suis capable de déterminer différents stades d'avancement de la mémoire épisodique dans un cas particulier comme celui-là, c'est parce que j'ai en tête un modèle de ce fonctionnement, qui me permet de faire la différence entre ces stades. Nous dirons par conséquent que les temps du passé font toujours référence à ce modèle naïf du fonctionnement de la mémoire 
épisodique, même dans les situations où le locuteur est réellement en train de faire usage de la mémoire épisodique.

Le dernier problème dont nous allons parler dans cette section est la fonction du passé composé. Dans la section 8 nous avons mis en évidence une différence importante entre ce temps et le passé simple, à savoir que lorsqu'on interprète un énoncé au passé simple on ne peut pas considérer la tâche suivie par la mémoire comme émanant d'un environnement mutuellement cognitif partagé par le locuteur et l'interlocuteur, une contrainte qui ne vaudrait pas pour le passé composé. Une manière d'expliquer cette différence serait de dire que le passé composé signale, non pas le fonctionnement de la mémoire, mais l'état mental qui en résulte, c'est-à-dire l'état d'avoir récupéré un souvenir épisodique au moment de l'énonciation ou avant ce moment. Cet état marque une étape finale du travail mnésique, la fin d'une recherche, ce qui signifie que l'énoncé suivant concernera l'état après une autre recherche faite d'après une nouvelle tâche. Ainsi entre chaque énoncé, le locuteur peut reprendre en considération l'environnement mutuellement cognitif qu'il partage avec son interlocuteur et choisir la prochaine tâche en fonction de cet environnement. Dans le cas du passé simple, les énoncés indiquent seulement des étapes franchies par la mémoire, mais pas son aboutissement. Le locuteur reste plongé dans son activité mnésique et ne l'ajuste pas par rapport à l'état cognitif de son interlocuteur.

\section{Conclusions}

L'article a seulement servi à montrer en quoi consisterait une hypothèse selon laquelle l'usage des temps du passé reflèterait différents modes de travail de la mémoire épisodique. Pour que cette hypothèse devienne une véritable approche dans le domaine de la sémantique du verbe, il faut tester sa capacité à expliquer des systèmes de temps du passé différents de celui du français. Il est également impératif de voir s'il est possible à partir de cette hypothèse d'expliquer d'autres temps grammaticaux comme le présent et le futur. 


\section{Minni og málfræðilegar pátíðir}

Greinin fjallar um hugsanleg tengsl á milli notkunar ólíkra málfræðilegra pátîđa í tungumáli og mismunandi starfshátta atburðarminnisins. Sú tilgáta er sett fram að pegar okkur er ljóst að segð miðlar minningu af liðnum atburði pá athugar sérhæf stöð tengd málskilningi hvort atburðarminnið leitaði að pessari minningu með ákveðið markmið að leiðarljósi eða hvort minningin kom nokkurn veginn ósjálfrátt til hugar talandans. Samkvæmt tilgátunni benda sumar málfræðilegar pátíðir sérstaklega á annað hvort minnisferli. Stöðin myndi líka athuga hvort pessi ábending um starfshátt atburðarminnisins samræmist innihaldi segðarinnar. Í greininni er tilgátan prófuð með tilliti til pátíðakerfis í frönsku. Bein tengsl væru annars vegar á milli passé simple (almenn pátîđ) og markvissa starfsháttar minnisins og hins vegar á milli imparfait (lýsingarpátíd) og ósjálfráđa starfsháttarins. Hlutverk plus-que-parfait (páli̊đin tî̉) væri að tilkynna að talandinn sé að íhuga atburð út frá öðrum atburði sem hann man eftir. Svo væri passé composé (núlíðin tî̉) tengd ástandinu sem felst í pví að hafa lokið minnisleit að liðnum atburði.

Lykilorð: pátîðakerfi frönskunnar, procedural meaning, minnið. 


\section{Memory and Past Tenses}

The paper investigates a possible link between the way we use different past tense categories and the way our memory recollects events from the past. The basic idea is that when it is contextually obvious that an utterance communicates an output from the episodic memory, a module gets activated which analyses whether the recollection followed a definite task or whether it acted spontaneously. Some verb tense categories would indicate specifically one of these two modes of recollection. But the module would also check also whether the utterance points to the same mode of recollection as the verb. The paper shows how this hypothesis works with the past tenses in French. A connection is proposed on one hand between imparfait and the spontaneous mode of recollection and on the other hand between passé simple and the goal-oriented mode of recollection. The function of the plus-que-parfait would be to indicate an extrapolation made by the locutor from an already recollected event, in order to stimulate the recollection of another event. The passé composé is associated with the state of having accomplished the recollection.

Keywords : French past tense categories, procedural meaning, memory. 


\section{BIBLIOGRAPHIE}

Apothéloz Denis et Combettes Bernard, «Saillance et aspect verbal : le cas du plusque-parfait ", Saillance. Aspects linguistiques et communicatifs de la mise en évidence dans un texte, 1/2011, Besançon: Presses universitaires de Franche-Comté, pp. 225-246.

Bastin Christine, «Le réseau cérébral par défaut : un repos qui n'en est pas un », Revue Neuropsychologie 10 (3)/2018, pp. 232-238.

Berthonneau Anne-Marie et Kleiber Georges, "Pour une nouvelle approche de l'imparfait. L'imparfait, un temps anaphorique méronomique »Langages 112/1993, pp. 55-73.

Berthonneau Anne-Marie et Kleiber Georges, « Pour une réanalyse de l'imparfait de rupture dans le cadre de l'hypothèse anaphorique méronomique », Cabiers de praxématique 32/1999, pp. 119-166.

Bres Jacques, L'imparfait dit narratif, Paris : CNRS, 2005.

Carston Robyn, "The heterogeneity of procedural meaning ", Lingua 175176/2016, pp. 154-166.

Dalgalian Gilbert et Malbert Daniel, Grammaire textuelle du français, Paris : Didier/ Hatier, 1989, p. 130, (traduction française de Harald Weinrich, Textgrammatik des französischen Sprache, Stuttgart : Klett, 1982).

Gerrans Philip, The measure of madness, 2014, Cambridge, MA : MIT Press, p. 17.

Heenen François, «Imparfait et Stéréotypes », Milli mála 7/2015, Reykjavík : Institut Vigdís Finnbogadóttir, pp. 121-149.

Heenen François, «Imparfait et modalité », Milli mála 8/2016, Reykjavík : Institut Vigdís Finnbogadóttir, pp. 93-117 .

Heenen François, «L'imparfait, un temps à deux procédures », Milli mála 7/2017, Reykjavík : Institut Vigdís Finnbogadóttir, pp. 97-120.

Hutto Daniel D., " Memory and narrativity », 2017, https://www.academia. edu/25340514/Memory_and_Narrativity.

Kamp Hans et Rohrer Christian, "Tense in texts ", Meaning, Use, and Interpretation of Language, 1983, éd. Bauerle R., Schwarze C. et von Stechow A., Berlin et New York, de Gruyter, pp. 250-269.

Leeman Danielle, «Le passé simple et son co-texte : examen de quelques distributions ", Langue française, Temps et co(n)texte, éd. Jacques Bres, 138/2003, pp. 20-34.

Luscher Jean-Marc et Sthioul Bertrand, « Emplois et interprétations du passé composé ", Cabiers de linguistique française, 18/1996, pp. 187-217.

Magliano Joseph P. et Schleich Michelle C., «Verb Aspect and Situation Models », Discourse Processes 29(2)/2000, pp. 83-112.

Mercier Hugo et Sperber Dan, The Enigma of Reason, 2017, Harvard University Press.

Moeschler Jacques, Grisot Cristina et Cartoni Bruno, « Jusqu'où les temps verbaux sont-ils procéduraux ? ", Nouveaux cabiers de linguistique française, 30/2012, pp. 119-139. 
Molendijk Arie L., Le passé simple et l'imparfait : une approche reichenbachienne, Amsterdam/ Atlanta, Rodopi, 1990.

Saussure Louis de et Sthioul Bertrand, «L'imparfait narratif : point de vue (et images du monde) », Cahiers de praxématique 32/1999, pp. 167-188; et Jacques Bres, Limparfait dit narratif, Paris : CNRS, 2005, pp. 51-62.

Saussure Louis de, "Quand le temps ne progresse pas avec le passé simple », Cabiers Chronos 6/2000, pp. 37-48, ici p. 37.

Saussure Louis de, Temps et pertinence, 2003, De Boeck/Duculot, Bruxelles.

Saussure Louis de, "Le passé composé en usage futur et la pertinence au présent », https://www.researchgate.net/profile/Louis_De_Saussure/publication/259422604_Le_passe_compose_en_usage_futur_et_la_pertinence_au_ present/links/546f7c990cf2d67fc031160c/Le-passe-compose-en-usage-futur-etla-pertinence-au-present.pdf

Sestieri Carlo, Corbetta Maurizio, Romani Gian Luca et Shulman Goldon L., «Episodic memory retrieval, parietal cortex, and the default mode network : functional and topographic analyses », 31/2011, pp. 4407-20.

Squire Larry R. et Zola Stuart M., « Structure and function of declarative and nondeclarative memory systems », Proc. Natl. Acad. Sci. USA, 93/1996, pp. 13515-13522.

Squire Larry R., " Memory and Brain Systems : 1969 -2009 », The Journal of Neuroscience, October 14/2009, pp. 12711-12716.

Tahara Izumi, Usage descriptif et usage interprétatif des temps du passé et des adverbes temporels dans le discours de fiction, Thèse de doctorat : Univ. Genève, 2004, DOI : 10.13097/archive-ouverte/unige :574, p. 233.

Tasmowski-De Ryck Liliane, « L'imparfait avec et sans rupture », Langue française, 67/1985, éd. n.c., lieu d'éd. n.c., pp. 59-77, ici p. 75.

Vetters Carl, "Passé simple, et imparfait : un couple mal assorti », Langue française, 100/1993, Temps et aspect dans la langue française, pp. 14-30.

Wilson Deirdre, "The conceptual-Procedural Distinction : Past, Present and Future ", Procedural Meaning : Problems and Perspectives, Current Research in Semantics / Pragmatics Interface 25/2011, éd. Victoria Escandell-Vidal, Manuell Leonetti et Aoife Ahern, pp. 3-31. 